\title{
ArcheoSciences
}

Revue d'archéométrie

\section{Les « turquoises culturelles » et la caractérisation par fluorescence de rayons X (XRF) in situ du disque de mosaïque de l'offrande 99 du Templo Mayor de México-Tenochtitlán}

The "cultural turquoise" and the XRF characterization in situ of the mosaic disc of the offering 99 from the Templo Mayor of México-Tenochtitlán

Kilian Laclavetine, Jose Luis Ruvalcaba-Sil, Christian Duverger et Emiliano Ricardo Melgar Tísoc

\section{OpenEdition}

Journals

Édition électronique

URL : https://journals.openedition.org/archeosciences/4269

DOI : $10.4000 /$ archeosciences.4269

ISBN : 978-2-7535-3691-3

ISSN : 2104-3728

Éditeur

Presses universitaires de Rennes

Édition imprimée

Date de publication : 30 novembre 2014

Pagination : 205-218

ISBN : 978-2-7535-3689-0

ISSN : 1960-1360

Référence électronique

Kilian Laclavetine, Jose Luis Ruvalcaba-Sil, Christian Duverger et Emiliano Ricardo Melgar Tísoc, « Les « turquoises culturelles » et la caractérisation par fluorescence de rayons X (XRF) in situ du disque de mosaïque de l'offrande 99 du Templo Mayor de México-Tenochtitlán », ArcheoSciences [En ligne], 38 | 2014, mis en ligne le 30 novembre 2016, consulté le 31 janvier 2022. URL : http://

journals.openedition.org/archeosciences/4269; DOI : https://doi.org/10.4000/archeosciences.4269 


\title{
Les « turquoises culturelles » et la caractérisation par fluorescence de rayons $\mathrm{X}(\mathrm{XRF})$ in situ du disque de mosaïque de l'offrande 99 du Templo Mayor de México-Tenochtitlán
}

\section{The "Cultural Turquoise" and the XRF Characterization in situ of the Mosaic Disc of the Offering 99 from the Templo Mayor of México-Tenochtitlán}

\author{
Kilian Laclavetinea, b, Jose Luis Ruvalcaba-SiL \\ Christian Duverger ${ }^{\text {b et Emiliano Ricardo Melgar Tísoc }}{ }^{c}$
}

\begin{abstract}
Résumé : Les Aztèques utilisèrent une quantité importante de minéraux bleus, généralement désignés comme turquoise pour confectionner, notamment, des offrandes. Notre étude propose de déterminer la composition chimique des minéraux bleus du disque de mosaïque de l'offrande 99 issu des fouilles du Templo Mayor de México-Tenochtitlán, ancienne capitale aztèque, et d'observer l'usage de ces minéraux dans la manufacture de cet objet. L'objectif est de proposer une première évaluation des différentes espèces minérales présentes à partir d'analyses in situ à l'aide de la fluorescence de rayons $\mathrm{X}$ en comparant les résultats obtenus à ceux d'une base de données minérale. La présente étude amène à préciser la théorie des « turquoises culturelles » proposée par Weigand et al. (1977) et reprise par Kim et al. (2003). Cette théorie suggère que la plupart des sociétés mésoaméricaines, au cours du temps, ont conféré une valeur symbolique à la couleur bleu-vert et ont employé des minéraux bleus, voire verts, pour confectionner, notamment, leurs objets d'offrandes. Toutefois, on peut aussi penser que la fabrication des objets pouvait s'effectuer sans préoccupation dans le choix de la matière première en mélangeant différentes espèces minérales de la famille des " turquoises culturelles ". Ce dernier point est discuté dans le cas des offrandes du Templo Mayor dont l'étude révèle l'absence de mélange intentionnel d'espèces minérales dans la confection de chaque objet et un usage quasi-exclusif des « turquoises minérales » comme matière première.
\end{abstract}

\begin{abstract}
The Aztecs used significant amounts of blue minerals, generally designated as turquoise, in their offerings. The aim of this work is to determine the chemical composition of blue minerals in the mosaic disc of the offering 99 discovered during the excavations of the Templo Mayor in México-Tenochtitlan, Aztecs former capital, and to study the usage of these minerals in the manufacture of this object. The objective is to propose a first evaluation of the different mineral species by performing in situ analyses using the X-ray fluorescence and comparing the obtained results to those of a mineral database. This study is based on the theory of the "cultural turquoise" established by Weigand and al. (1977) and resumed by Kim et al. (2003). It suggests that most of the Mesoamerican societies, along times, conferred a symbolic value to the green blue color and employed blue and green minerals to prepare offerings. Nevertheless, one can also think that the manufacture of the objects was carried out without giving a lot of importance to the choice of the raw material when mixing various mineral species from "cultural turquoise". This last point is discussed in the case of the offerings of the Templo Mayor. The study reveals the lack of mixture of mineral species in the fabrication process of the analyzed objects and an almost exclusive usage of "chemical turquoise" as raw material.
\end{abstract}

Mots clés : Aztèque, Mésoamérique, Offrande, Templo Mayor, Turquoise, XRF.

Keywords: Aztec, Mesoamerica, Offering, Templo Mayor, Turquoise, XRF.

\footnotetext{
anstituto de Física, Universidad Nacional Autónoma de México, Mexico, Mexique. (kilian@us.es) (sil@fisica.unam.mx)

${ }^{\mathrm{b}}$ Centre de recherche sur l'Amérique préhispanique, EA 3551, Écoles des Hautes Études en Sciences Sociales, Paris, France. (Christian.Duverger@ehess.fr)

b Museo del Templo Mayor, Instituto Nacional de Antropología e Historia, Mexico, Mexique. (anubismarino@gmail.com)
} 


\section{INTRODUCTION}

Les Aztèques (Mexicas) utilisèrent une quantité importante de minéraux bleus, généralement désignés comme "turquoise ", dans la confection d'objets (Saville, 1922). Les fouilles du Templo Mayor de México-Tenochtitlán, l'antique capitale mexica, ont révélé de nombreux objets d'offrandes couverts de mosaïques de minéraux bleus (Lopez Lujan, 1993; Matos Moctezuma, 1994). Cette étude vise à déterminer la composition chimique des minéraux bleus constituant ces offrandes et à observer l'usage de ces minéraux dans l'élaboration de ces artefacts. Ce projet est le fruit d'une collaboration entre le Musée du Templo Mayor, l'Institut National d'Anthropologie et d'Histoire du Mexique (INAH) et l'Institut de Physique de l'Université Nationale Autonome de Mexico (IFUNAM).

La question de la provenance de ces minéraux est depuis longtemps au centre des études archéométriques menées sur ces objets archéologiques (Weigand et al., 1977; Weigand et Harbottle, 1993; Mathien, 2001; Kim et al., 2003). Ce travail propose de développer une étape préliminaire indispensable à une étude de provenance, à savoir, une première évaluation des différentes espèces minérales susceptibles d'être employées dans la confection de ces objets rituels. Cette première approximation effectuée sur les offrandes religieuses permet d'aborder d'autres problématiques concernant, par exemple, la chaîne opératoire de fabrication de ces objets d'une grande valeur symbolique, ou encore, la sémantique des pierres dans le langage religieux aztèque. Cette dernière question anthropologique a récemment été étudiée et discutée du point de vue historique et linguistique (Izeki, 2008). Cependant, elle n’a jamais été abordée, jusqu’à présent, d'un point de vue archéométrique. D'autre part, cette étude propose de préciser la théorie des «turquoises culturelles " proposée par Weigand et al. (1977) et reprise par Kim et al. (2003). Cette théorie suggère que la plupart des sociétés mésoaméricaines, au cours du temps, ont conféré une valeur symbolique à la couleur bleu-vert et ont employé des minéraux bleus, voire verts, pour confectionner, notamment, leurs objets d'offrandes. Dans le cadre de cette théorie, on peut penser que la fabrication des objets pouvait s'effectuer sans préoccupation dans le choix de la matière première en mélangeant différentes espèces minérales appartenant à la famille des " turquoises culturelles ». C'est ce dernier point que nous aimerions étudier et discuter dans le cas des offrandes du Templo Mayor. L'objectif de ce travail est d'apporter une première évaluation sur l'éventuelle existence de mélange de ces espèces dans la confection de chaque objet.
La présente contribution est dédiée à la présentation des résultats de l'étude in situ du disque de mosaïque de l'offrande 99 de la collection du Musée du Templo Mayor par la mise en ouvre de mesures de fluorescence de rayons X (XRF) de manière non destructive. L'étude consiste en une première approximation concernant la distinction indirecte des espèces minérales via XRF en s'appuyant sur une base de données développée dans le cadre de ce projet. Il s'agit de la première étude archéométrique par XRF effectuée sur cet objet unique. Les résultats seront comparés à ceux obtenus dans nos études précédentes (Laclavetine, 2008; Laclavetine et al., sous presse).

\section{ConteXte archeologiQue}

México-Tenochtitlán fut la capitale des Aztèques de 1325 à 1519 après Jésus-Christ. Une des plus grandes métropole de Mésoamérique s'est développée autour de son centre cérémonial au milieu duquel s'élevait le Templo Mayor. Cet ensemble architectonique était non seulement le cœur géographique de la cité mais aussi le cœur religieux, politique et social de la société mexica. Ce lieu hautement sacré a reçu de nombreuses offrandes qui ont été découvertes lors des fouilles du Templo Mayor dans le centre historique de la capitale moderne, Mexico (Lopez Lujan, 1993; Matos Moctezuma, 1994). Une partie de ces offrandes est composée d'objets incrustés et de mosaïques de minéraux bleus, désignés traditionnellement comme turquoise.

Le Musée du Templo Mayor conserve, expose et étudie le matériel archéologique découvert pendant les différentes phases de fouille du projet intitulé "Proyecto Templo Mayor " (Lopez Lujan, 1993). Les vestiges du Templo Mayor sont une source précieuse d'informations puisque les dates des différentes étapes de construction sont connues (tableau 1). De plus, la nature hautement symbolique des offrandes enfouies autour de l'édifice pyramidal, au cours des cérémonies organisées par l'appareil politique et religieux, offre un corpus de choix pour l'étude de la symbolique de ces objets ainsi que de la matière première qui les compose (Lopez Lujan, 1993; Duverger, 2007).

Le disque de l'offrande 99 est une mosaïque composée de plus de 15000 tesselles de tailles qui vont de l'ordre du millimètre au centimètre (figure 1). Les tesselles présentent des nuances de couleurs variant du blanc au bleu en passant par le vert. On peut observer onze tonalités, d'après les valeurs établies dans la table de Munsell (Ruvalcaba et al., 2005). Les tesselles sont de formes variées : disques, triangles, carrés, rectangles, trapèzes, polygones, etc. La restauration minutieuse de cet objet a abouti à un disque de 28 centimètres 


\begin{tabular}{|l|c|c|c|c|}
\hline \multicolumn{1}{|c|}{$\begin{array}{c}\text { Étapes de } \\
\text { construction }\end{array}$} & Années & Événements & Offrandes analysées & $\begin{array}{c}\text { Nombre de tesselles analysées par } \\
\text { offrande }\end{array}$ \\
\hline Étape I & $1325-1375$ & Fondation de Tenochtitlan & & \\
\hline Étape II & $1375-1426$ & $\begin{array}{c}\text { Règnes d'Acamapichtli, d'Huit- } \\
\text { zilíhuitl et de Chimalpopoca }\end{array}$ & & \\
\hline Étape III & $1427-1440$ & Règne d'Itzcóatl & & 60 et 59 \\
\hline Étape IVa & $1440-1469$ & Règne de Moctezuma I & Offrande 48 et Xiuhcoatl & 55 et 46 \\
\hline Étape IVb & $1469-1481$ & Règne d'Axayácatl & Offrandes 6 et 17 & 31 \\
\hline Étape V & $1481-1486$ & Règne de Tízoc & Offrande K & \\
\hline Étape VI & $1486-1502$ & Règne d'Ahuízotl & Offrande 99 & 442 \\
\hline Étape VII & $1502-1520$ & Règne de Moctezuma II & & \\
\hline
\end{tabular}

Tableau 1 : Liste chronologique des offrandes du Templo Mayor de México-Tenochtitlán contenant les objets de turquoise analysés. Table 1: Chronological list of the offerings of the Templo Mayor of México-Tenochtitlán containing the turquoise analyzed objects.

Figure 1 : Disque de mosaïque de l'offrande 99 du Templo Mayor.

Figure 1: Mosaic disc of the offering 99 from the Templo Mayor.

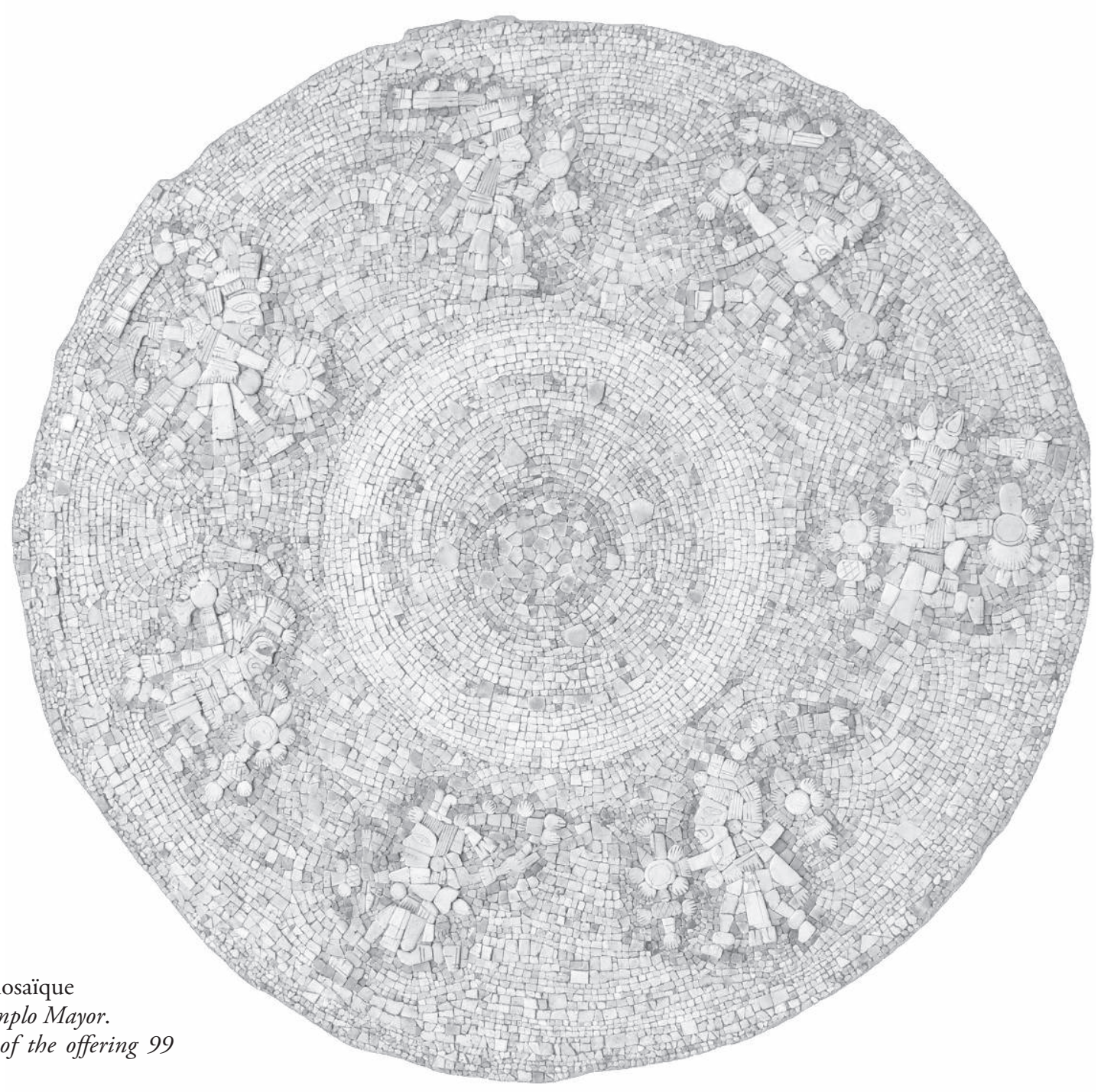

ArCheoSCiences, revue d'archéométrie, 38, 2014, p. 205-218 
de diamètre. En son centre, se situe un premier disque de 8,5 centimètres de diamètre de tesselles foncées bordé d'une bande plus claire de 1,5 centimètre de large, le tout encerclé par une bande de 8 centimètres de large dans laquelle sont représentés sept personnages masculins et divinités (Ruvalcaba et al., 2005).

\section{LES TURQUOISES « CULTURELLES »}

Il existe une théorie selon laquelle les Mésoaméricains auraient utilisé, pour confectionner leurs objets, des minéraux bleus et verts parmi lesquels figurait la turquoise. Ce groupe de minéraux fût d'abord nommé " pierre bleu-vert ». L'expression " turquoise culturelle " (cultural turquoise, en anglais) fût ensuite proposée pour désigner cet ensemble théorique (Weigand et al., 1977; Harbottle et Weigand, 1992). Cette expression englobe différents minéraux bleus et verts dont l'aspect visuel est proche de la turquoise. Elle est à distinguer de l'expression " turquoise minéralogique " (chemical turquoise, en anglais) qui désigne le minéral turquoise à proprement parler avec sa composition chimique et sa structure cristalline caractéristiques.

Le terme " turquoise " désigne aussi une nuance de bleu qui peut prêter à confusion. En effet, la palette de coloration de ce minéral n'est pas seulement limitée au bleu turquoise. Elle s'étale du bleu au vert de gris en passant par bleu ciel, bleu vert, vert, ou encore, blanc. Qu'il s'agisse de la classification de Dana (Groupe Dana 42.9.3) ou de celle de Struntz (« Groupe Turquoise "VII/D.15), la turquoise appartient à une famille de six minéraux (tableau 2) qui se construisent suivant la même structure minérale (ce qui peut compliquer leur différenciation physico-chimique) et dont la formule générale est :

$\mathrm{AB}_{6}\left(\mathrm{PO}_{4}\right)_{X}\left[\mathrm{PO}_{3}(\mathrm{OH})\right]_{4-\mathrm{X}}(\mathrm{OH})_{8} \cdot 4\left(\mathrm{H}_{2} \mathrm{O}\right)$

avec $\mathrm{A}^{2+}: \mathrm{Cu}^{2+}, \mathrm{Ca}^{2+}, \mathrm{Zn}^{2+}, \mathrm{Fe}^{2+}$ et $\mathrm{B}^{3+}: \mathrm{Al}^{3+}, \mathrm{Fe}^{3+}$.

Dans la suite de cet article, l'expression «éléments secondaires » est employée pour désigner les éléments atomiques qui appartiennent aux autres espèces minérales $\mathrm{du}$ " Groupe Turquoise ». Dans les tesselles analysées comme dans les turquoises de référence, les présences du fer $(\mathrm{Fe})$, du calcium (Ca) ou du zinc $(\mathrm{Zn})$ peuvent s'expliquer par des insertions du « Groupe Turquoise ». Pour autant, l'expression « turquoise minéralogique ", dans cet article, désigne l'ensemble des espèces minérales appartenant au "Groupe Turquoise ", à savoir, en plus de la turquoise, la faustite, la chalcosidérite, la planérite, la coeruleolastite et l'aheylite.

D’après une liste proposée par Kim et al. (2003), les " turquoises culturelles » incluent plus particulièrement la turquoise, l'azurite, la chrysocolle, la malachite, diverses combinaisons à base de quartz, avec ou sans calcite; viennent s'ajouter, dans un second temps, l'amazonite et le lapis lazuli. On aurait assigné un même rôle culturel ou cultuel, au cours du temps, à cet ensemble. Toutefois, cet aspect de la sémantique des pierres n'a jamais été traité d'un point de vue archéométrique.

\section{DéVeloppement EXPERIMENTAL}

Les offrandes du Templo Mayor sont des objets uniques et précieux pour le patrimoine archéologique et culturel mexicain. Dans le cas des offrandes comportant des minéraux bleus, il n'est pas envisageable de procéder à des analyses micro-destructives ou micro-invasives. Il est donc nécessaire d'opter pour une technique pouvant être appliquée in situ, capable de produire des résultats via des analyses non destructives et non invasives et permettant d'étudier un grand nombre de tesselles.

La fluorescence de rayons $\mathrm{X}$ est connue pour ces nombreuses applications en archéométrie depuis de nombreuses années (notamment dans des études de provenance de turquoises étasuniennes : Mathien et al. [1992] ; Ronzio et al. [1967] ; Salmon et al. [1962]). La technique XRF est une méthode de caractérisation basée sur les interactions des rayons X avec la matière (Moens et al., 2000; Garrison,

\begin{tabular}{|l|c|c|}
\hline Nom & Formule chimique & Endmember $(\mathrm{A}, \mathrm{B})$ \\
\hline Turquoise & $\mathrm{CuAl}_{6}\left(\mathrm{PO}_{4}\right)_{4}(\mathrm{OH})_{8} \cdot 4\left(\mathrm{H}_{2} \mathrm{O}\right)$ & $(\mathrm{Cu}, \mathrm{Al})$ \\
\hline Coeruleolactite & $\mathrm{Ca}_{1-\mathrm{C}} \mathrm{Cu}_{\mathrm{x}} \mathrm{Al}_{6}\left(\mathrm{PO}_{4}\right)_{4}(\mathrm{OH})_{8} \cdot 4-5\left(\mathrm{H}_{2} \mathrm{O}\right)$ & $(\mathrm{Ca} / \mathrm{Cu}, \mathrm{Al})$ \\
\hline Faustite & $\mathrm{Zn}_{1-\mathrm{x}} \mathrm{Cu}_{\mathrm{x}} \mathrm{Al}_{6}\left(\mathrm{PO}_{4}\right)_{4}(\mathrm{OH})_{8} \cdot 4\left(\mathrm{H}_{2} \mathrm{O}\right)$ & $(\mathrm{Zn} / \mathrm{Cu}, \mathrm{Al})$ \\
\hline Chalcosidérite & $\mathrm{CuFe}_{6}{ }_{6}\left(\mathrm{PO}_{4}\right)_{4}(\mathrm{OH})_{8} \cdot 4\left(\mathrm{H}_{2} \mathrm{O}\right)$ & $(\mathrm{Cu}, \mathrm{Fe})$ \\
\hline Aheylite & $\mathrm{Fe}^{2+}{ }_{1-x-y} \mathrm{Zn}_{\mathrm{x}} \mathrm{Al}_{6}\left(\mathrm{PO}_{4}\right)_{4-z}\left(\mathrm{PO}_{3} \mathrm{OH}\right)_{z}(\mathrm{OH})_{8} \cdot 4+\mathrm{y}\left(\mathrm{H}_{2} \mathrm{O}\right)$ & $(\mathrm{Fe} / \mathrm{Zn}, \mathrm{Al})$ \\
\hline Planérite & $\mathrm{Al}_{6}\left(\mathrm{PO}_{4}\right)_{2}\left(\mathrm{PO}_{3} \mathrm{OH}\right)_{2}(\mathrm{OH})_{8} \cdot 4\left(\mathrm{H}_{2} \mathrm{O}\right)$ & $(\mathrm{Cu} / \mathrm{Ca}, \mathrm{Al})$ \\
\hline
\end{tabular}

Tableau 2 : Minéraux composants le "groupe turquoise".

Table 2: Minerals composing the "turquoise group". 
2003; Demortier, 2005). L'IFUNAM a développé une grande expérience dans les analyses in situ de documents historiques avec l'équipement SANDRA (Zetina et al., 2009). Il a déjà permis l'étude d'une grande variété de pièces et d'objets de musée et d'œuvres d'art comme des statues, des peintures sur chevalet, des céramiques décorées, de la soie, des documents antiques, des bijoux et des pierres vertes (Ruvalcaba et al., 2011; Ruvalcaba et al., 2010a). SANDRA est équipé d'une source de rayons $\mathrm{X}$ (tube de molybdène, 75W, modèle 75011, Oxford Instruments), d'un détecteur de rayons X de type Si-PIN (modèle XR100CR, Amptek), d'un générateur haute tension, d'une caméra numérique pour conserver une image des zones analysées ainsi que d'un ordinateur pour l'acquisition des spectres XRF et le stockage des photographies (Ruvalcaba et al., 2010b).

Néanmoins, l'emploi de cette technique comme une première approximation dans le but de distinguer différentes espèces minérales est inédit. Cette méthodologie a été présentée et discutée dans un travail préalable (Laclavetine et al., sous presse). La méthode s'appuie sur la distinction indirecte des espèces minérales composant les objets archéologiques en comparant les spectres XRF à ceux d'une base de données minérale.

La première étape a consisté à la création d'une base de données de minéraux bleus et verts dont l'espèce minérale a été identifiée par différentes méthodes et notamment par diffraction de rayons X (DRX). Cette collection minérale de références a été analysée par spectroscopie infrarouge (IR), par XRF, par émission de rayons X induite par particules (PIXE), par DRX, par spectroscopie Raman, par ionoluminescence, par colorimétrie et par radiométrie. Cette base de données a été constituée grâce à la collaboration de la Collection de Minéraux de l'Université Pierre et Marie Curie, du Centre de Spectroscopie infrarouge du Muséum National d'Histoire Naturelle, de l'Institut de Géologie de l'Université National Autonome de Mexico (Instituto de Geología de la UNAM, Mexico) et de l'Institut de Physique de l'UNAM (IFUNAM, Mexico).
Les minéraux composant cette collection sont présentés dans le tableau 3. La sélection des minéraux pour cette première étude a été faite en fonction des minéraux les plus susceptibles d'être rencontrés dans les offrandes analysées. Il s'agit des espèces minérales référencées comme étant présentes dans les offrandes mésoaméricaines et les artefacts du Sud-ouest étasunien dans les études précédentes effectuées depuis 1922 à nos jours, résumées dans l'article de Kim et al. de 2003. Il est évident que si, dans le futur, une étude démontre la présence d'autres minéraux dans les offrandes, notre recherche devra être révisée en intégrant les nouvelles espèces minérales en question. Etant donné qu'il n'existe pas de base de données complète, ni de collection de matériel de référence exhaustifs, ni de prospections des sources minérales adéquates, c'est le contexte archéologique qui dirige notre recherche en termes de matière première, de minéraux, de sources, de technologie, etc. En plus de l'état de l'art, le choix des espèces minérales sélectionnées pour former cette première base de données prend en considération trois autres aspects : la présence d'une couleur opaque, une dimension spécifique (minéraux supérieurs à $2 \mathrm{~mm}$ de côté) et une dureté suffisante. La considération du type de matériaux qui a pu être employé pour produire les tesselles est importante et discriminante.

En théorie, en se basant sur les formules chimiques des différents minéraux sélectionnés, sur la couleur parfois spécifique des espèces minérales et sur les capacités de détection de la $\mathrm{XRF}$, il est possible de les distinguer. En effet, tous les minéraux sont composés de cuivre $(\mathrm{Cu})$ mis à part l'amazonite et le lapiz lazuli (tableau 3). L'azurite et la malachite se différencient par l'absence d'autres éléments atomiques plus lourds que l'aluminium; la turquoise par la présence de phosphore (P) ; la chrysocolle par la présence de silicium (Si). Par la suite, l'azurite bleue se distingue visuellement de la malachite verte. Dans le cas des minéraux ne contenant pas de $\mathrm{Cu}$, l'amazonite se distingue par la présence de potassium (K) tandis que le lapiz lazuli, par la présence de $S$ et de Ca.

\begin{tabular}{|l|c|c|c|}
\hline Nom & Formule chimique & Eléments détectables par XRF & Dureté (Echelle de Mohs) \\
\hline Turquoise & $\mathrm{CuAl}_{6}\left(\mathrm{PO}_{4}\right)_{4}(\mathrm{OH})_{8} \cdot 4\left(\mathrm{H}_{2} \mathrm{O}\right)$ & $\mathrm{Al}, \mathrm{P}, \mathrm{Cu}$ & $5-6$ \\
\hline Chrysocolle & $\mathrm{Cu}_{2-\mathrm{x}} \mathrm{Al}_{\mathrm{x}} \mathrm{H}_{2-\mathrm{x}}\left(\mathrm{Si}_{2} \mathrm{O}_{5}\right)(\mathrm{OH})_{4} \cdot x\left(\mathrm{H}_{2} \mathrm{O}\right)$ & $\mathrm{Al}, \mathrm{Si}, \mathrm{Cu}$ & $2,5-3,5$ \\
\hline Azurite & $\mathrm{Cu}_{3}\left(\mathrm{CO}_{3}\right)_{2}(\mathrm{OH})_{2}$ & $\mathrm{Cu}$ & $3,5-4$ \\
\hline Malachite & $\mathrm{Cu}_{2}\left(\mathrm{CO}_{3}\right)(\mathrm{OH})_{2}$ & $\mathrm{Cu}$ & $3,5-4$ \\
\hline Amazonite (Microcline) & $\mathrm{KAlSi}_{3} \mathrm{O}_{8}$ & $\mathrm{Al}, \mathrm{Si}, \mathrm{K}$ & Microcline $: 6-6,5$ \\
\hline Lapiz Lazuli (Lazurite) & $\mathrm{Na}_{3} \mathrm{Ca}_{2}\left(\mathrm{Al}_{3} \mathrm{Si}_{3} \mathrm{O}_{12}\right) \mathrm{S}$ & $\mathrm{Al}, \mathrm{Si}, \mathrm{S}, \mathrm{Ca}$ & Lazurite $: 5-5,5$ \\
\hline
\end{tabular}

Tableau 3 : Minéraux composant la base de données de référence avec leur formule chimique, les éléments détectables par XRF et la dureté estimée sur l'échelle de Mohs.

Table 3: Minerals composing the reference database with their chemical formula, the elements detectable by XRF and the hardness on Mohs scale. 
En pratique, une étude des possibilités et des limites de la distinction d'espèces minérales à partir des seuls spectres XRF dans le cas précis de notre collection minérale a été effectuée. La tension d'alimentation du tube a été fixée à $30 \mathrm{kV}$ pour une intensité de $300 \mu \mathrm{A}$. Le temps d'acquisition était de 120 secondes par spectre. Les tableaux $4 a$ et $4 \mathrm{~b}$ présentent les résultats du dépouillement des spectres $\mathrm{XRF}$ obtenus. On retrouve tous les éléments caractéristiques attendus pour chaque espèce minérale. Les minéraux ne contenant pas de $\mathrm{Cu}$ se distinguent parfaitement des minéraux cuivrés. L'azurite et la malachite se différencient bien par la présence massive de $\mathrm{Cu}$. On est en mesure de distinguer la chrysocolle contenant du Si de la turquoise contenant du P. Il est à noter que les spectres XRF de la turquoise présentent systématiquement du $\mathrm{Ca}$, du Fe et du $\mathrm{Zn}$ qui participent à la structure des autres minéraux du "Groupe Turquoise ». Mis à part le Ca qui est également présent dans les chrysocolles, les éléments Fe et $\mathrm{Zn}$, absents des chrysocolles, peuvent être considérés comme des éléments caractéristiques des « turquoises minéralogiques ». Cependant, cette constatation confirme que la technique XRF ne permet pas de distinguer les différentes espèces minérales du «Groupe Turquoise ». Cette observation, très importante au niveau de la définition de l'espèce minérale, n'a pas d'importance pour la problématique archéologique de notre étude. Finalement, il est important de préciser que la présence ou non d'aluminium n'est pas considérée comme discriminante car, étant un élément léger, l'aluminium peut être en concentration inférieure à la limite de détection. C'est à partir de ces critères et en tenant compte de ces considérations que nous distinguerons les minéraux bleu-vert.

La discussion de cette méthodologie originale conclue que la fluorescence de rayons $\mathrm{X}$, bien que non adaptée à la différenciation des structures cristallines, permet une première approche des espèces minérales de notre corpus de référence. En combinant l'observation visuelle (couleur du minéral dans le cas de la malachite (vert) et de l'azurite (bleu), par exemple) aux spectres XRF, il est possible de différencier les minéraux de référence.

\section{Methodologie}

Le disque de l'offrande 99 du Musée du Templo Mayor a été étudié, de manière non-destructive, in situ, à l'aide du dispositif SANDRA. Les conditions expérimentales ont été sélectionnées de façon à favoriser la lecture des éléments légers (Al, Si, P, S). La tension d'alimentation du tube a été fixée à $30 \mathrm{kV}$ pour une intensité de $300 \mu \mathrm{A}$. Le temps d'acquisition était de 120 secondes par spectre. Au total,

\begin{tabular}{|c|c|c|c|c|c|c|c|}
\hline Espèce minérale & $\mathrm{Al}-\mathrm{K} \alpha$ & Si-K $\alpha$ & $\mathrm{P}-\mathrm{K} \alpha$ & S-K $\alpha$ & K-Ko & $\mathrm{Ca}-\mathrm{K} \alpha$ & $\mathrm{Cu}-\mathrm{K} \alpha$ \\
\hline Turquoise & $0.21 \pm 0.01$ & - & $1.10 \pm 0.01$ & - & $0.14 \pm 0.01$ & $0.07 \pm 0.01$ & $90.32 \pm 0.01$ \\
\hline Chrysocolle & $0.04 \pm 0.01$ & $0.24 \pm 0.01$ & - & - & - & $1.67 \pm 0.01$ & $97.87 \pm 0.01$ \\
\hline Azurite & $0.03 \pm 0.01$ & $0.02 \pm 0.01$ & - & $0.1 \pm 0.01$ & $0.05 \pm 0.01$ & $0.27 \pm 0.01$ & $95.25 \pm 0.01$ \\
\hline Malachite & $0.02 \pm 0.01$ & - & - & - & - & - & $99.98 \pm 0.01$ \\
\hline Amazonite & $0.58 \pm 0.01$ & $5.22 \pm 0.01$ & - & - & $39.49 \pm 0.01$ & - & - \\
\hline Lapiz Lazuli & $0.4 \pm 0.01$ & $3.72 \pm 0.01$ & - & $1.98 \pm 0.01$ & $1.05 \pm 0.01$ & $76.39 \pm 0.01$ & - \\
\hline
\end{tabular}

Tableau 4a : Aires des pics caractéristiques normalisées (avec leurs erreurs statistiques) des minéraux de référence avec, en gras, les éléments constitutifs de la structure cristalline principale.

Table 4a: Normalized areas of the characteristic peaks (with their statistical errors) of the reference minerals with, in bold, the components of the major crystalline structure.

\begin{tabular}{|l|c|c|c|c|c|c|c|c|}
\hline Espèce minérale & $\mathrm{Cl}-\mathrm{K} \alpha$ & $\mathrm{Ti}-\mathrm{K} \alpha$ & $\mathrm{Mn}-\mathrm{K} \alpha$ & $\mathrm{Fe}-\mathrm{K} \alpha$ & $\mathrm{As}-\mathrm{K} \alpha$ & $\mathrm{Rb}-\mathrm{K} \alpha$ & $\mathrm{Sr}-\mathrm{K} \alpha$ & $\mathrm{Pb}-\mathrm{L} \alpha$ \\
\hline Turquoise & - & $0.28 \pm 0.01$ & - & $7.58 \pm 0.01$ & $0.31 \pm 0.01$ & - & - \\
\hline Chrysocolle & - & - & - & - & - & - & $0.17 \pm 0.01$ \\
\hline Azurite & - & $0.02 \pm 0.01$ & $0.05 \pm 0.01$ & $1.01 \pm 0.01$ & $0.94 \pm 0.01$ & - & - \\
\hline Malachite & - & - & - & - & - & - & - \\
\hline Amazonite & - & - & - & $1.07 \pm 0.01$ & - & $30.14 \pm 0.01$ & - \\
\hline Lapiz Lazuli & $0.44 \pm 0.01$ & $1.96 \pm 0.01$ & $1.62 \pm 0.01$ & $9.52 \pm 0.01$ & - & - & $2.91 \pm 0.01$ \\
\hline
\end{tabular}

Tableau 4b : Aires des pics caractéristiques normalisées (avec leurs erreurs statistiques) des minéraux de référence.

Table 4b: Normalized areas of the characteristic peaks (with their statistical errors) of the reference minerals. 
443 tesselles du disque de mosaïque de l'offrande 99 ont été analysées (figures 2 à 9). Ce corpus représente presque $3 \%$ des tesselles composant le disque.

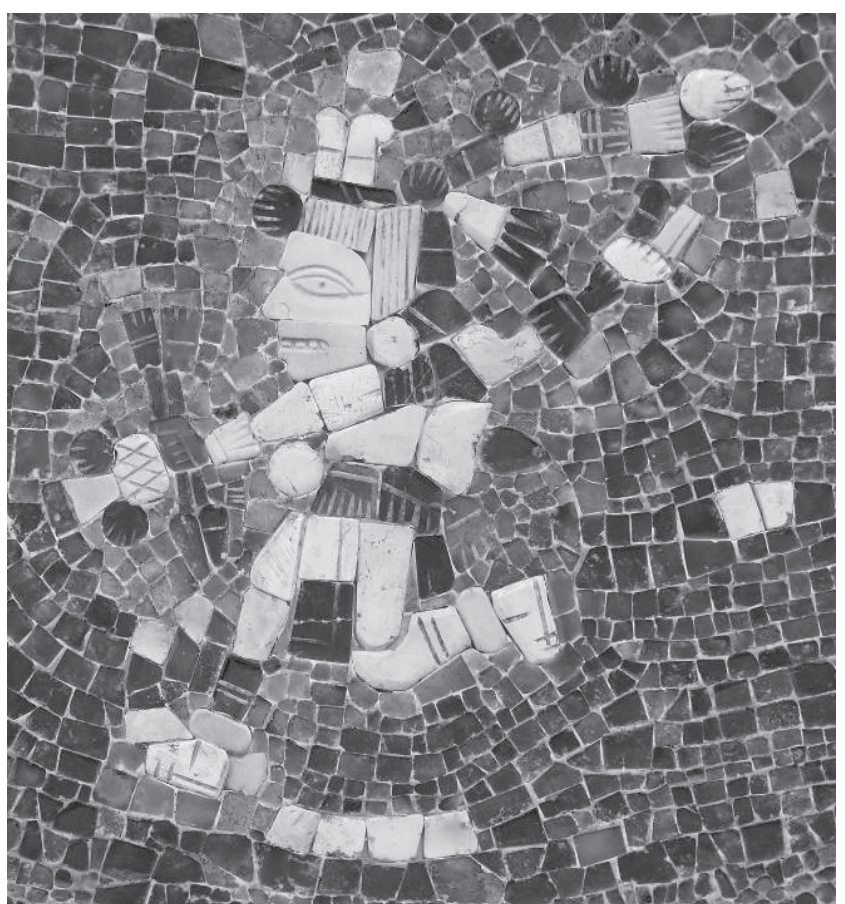

Figure 2 : Détail de l'offrande 99 avec, en clair, les tesselles analysées par XRF ( 49 mesures).

Figure 2: Detail of the offering 99. In light gray, the tesserae analyzed by XRF ( 49 measurements).

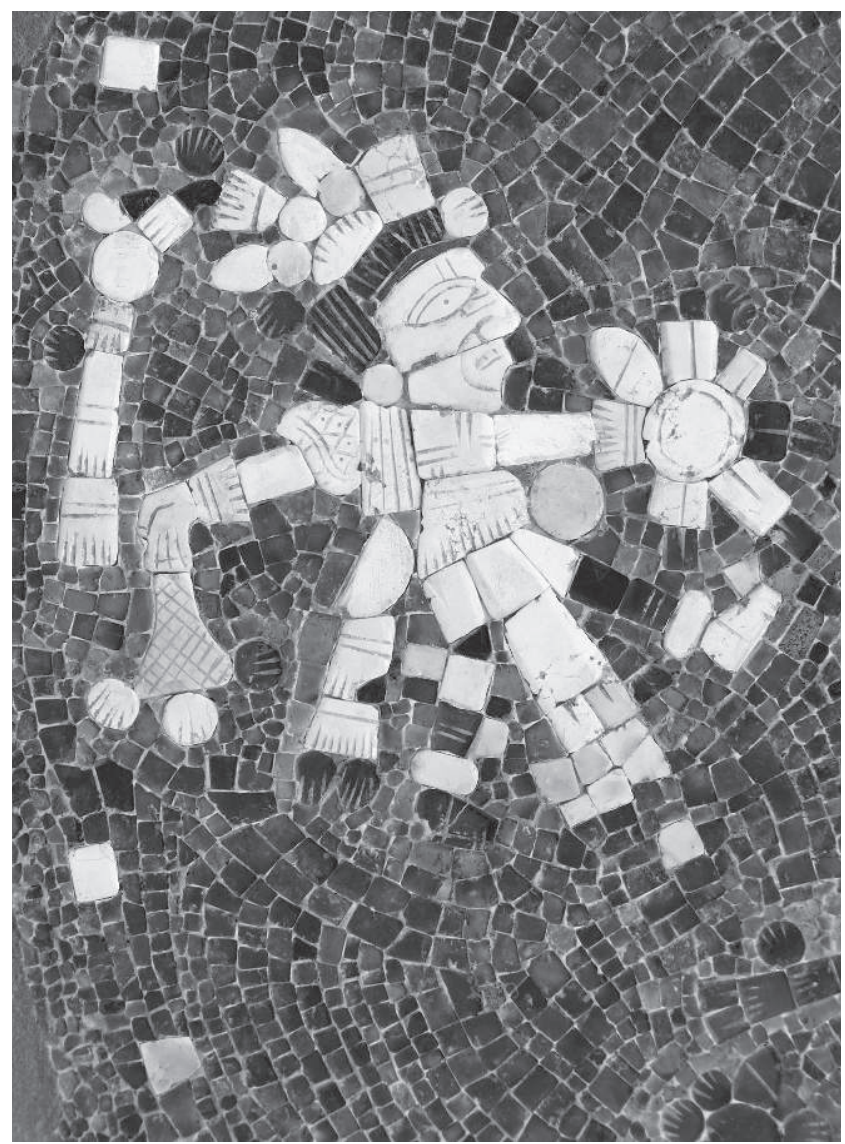

Figure 3 : Détail de l'offrande 99 avec, en clair, les tesselles analysées par XRF (63 mesures).

Figure 3: Detail of the offering 99. In light gray, the tesserae analyzed by XRF (63 measurements).
Figure 4 : Détail de l'offrande 99 avec, en clair, les tesselles analysées par XRF (67 mesures).

Figure 4: Detail of the offering 99. In light gray, the tesserae analyzed by XRF (67 measurements).

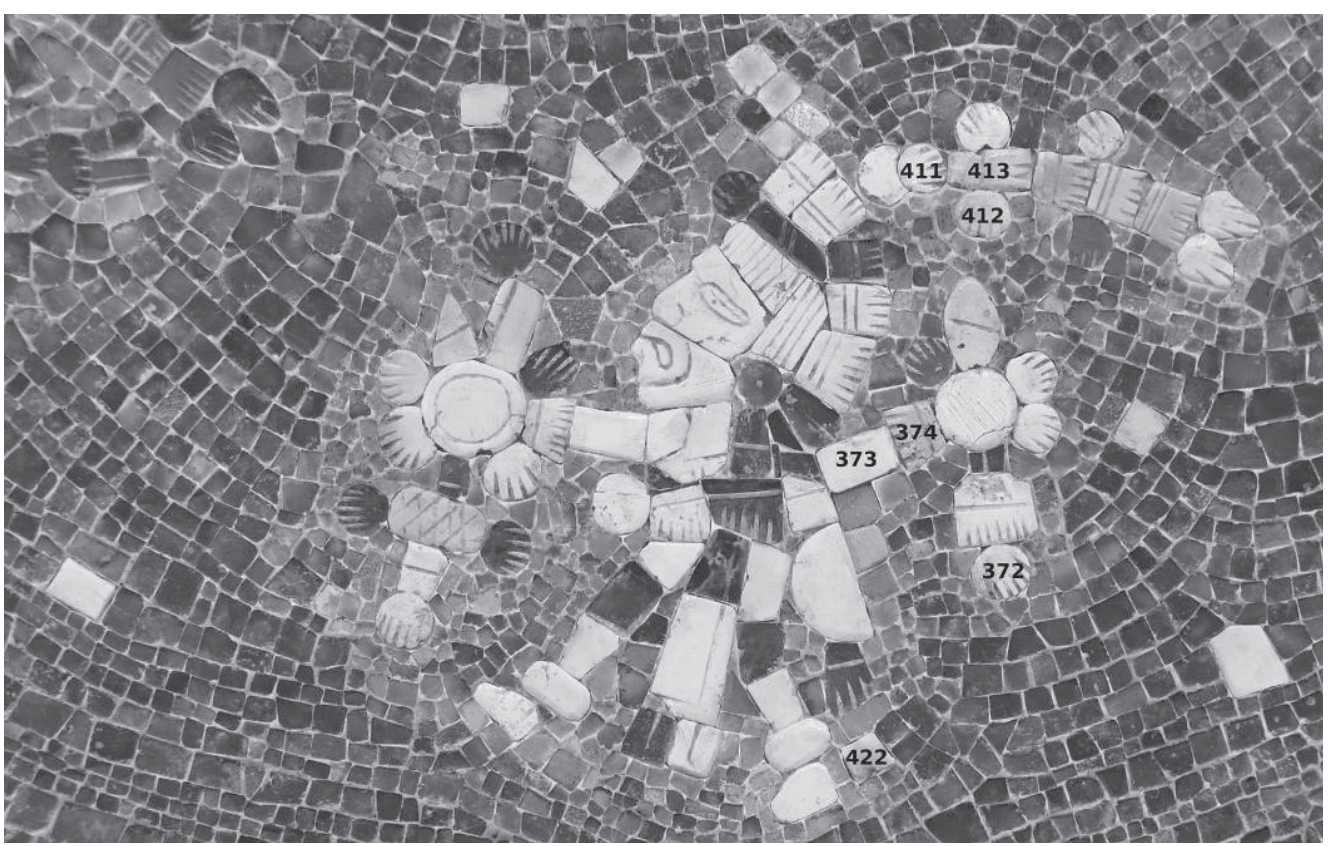




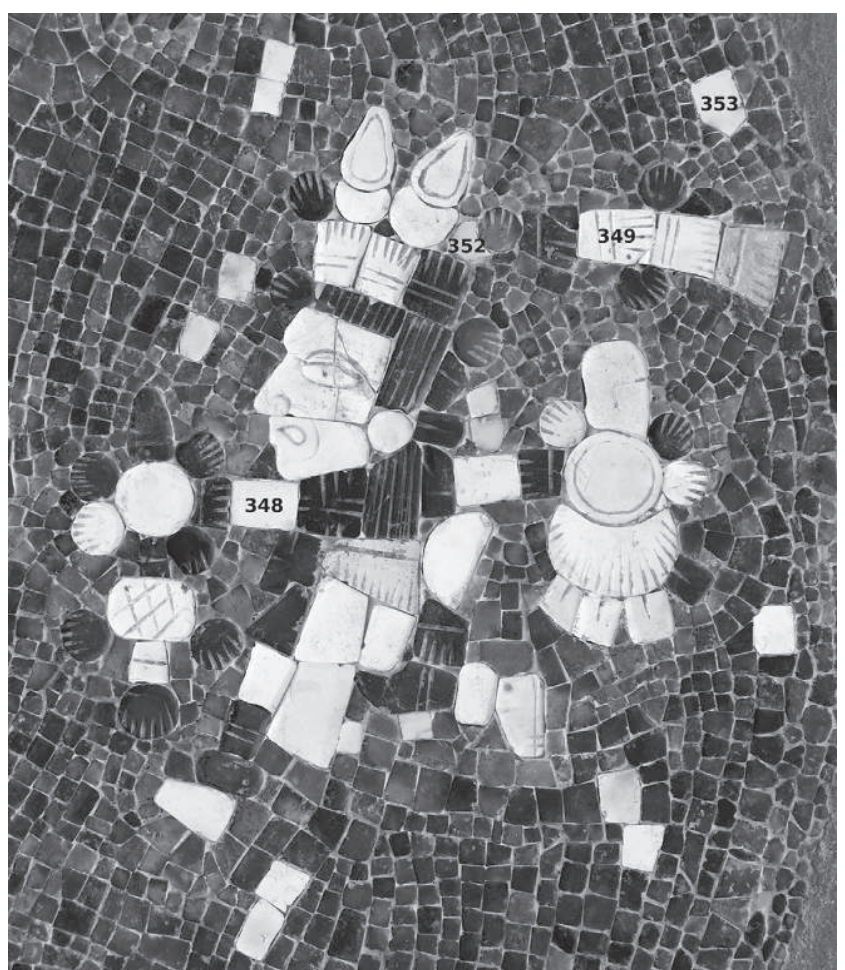

Figure 5 : Détail de l'offrande 99 avec, en clair, les tesselles analysées par XRF (50 mesures).

Figure 5: Detail of the offering 99. In light gray, the tesserae analyzed by XRF (50 measures).

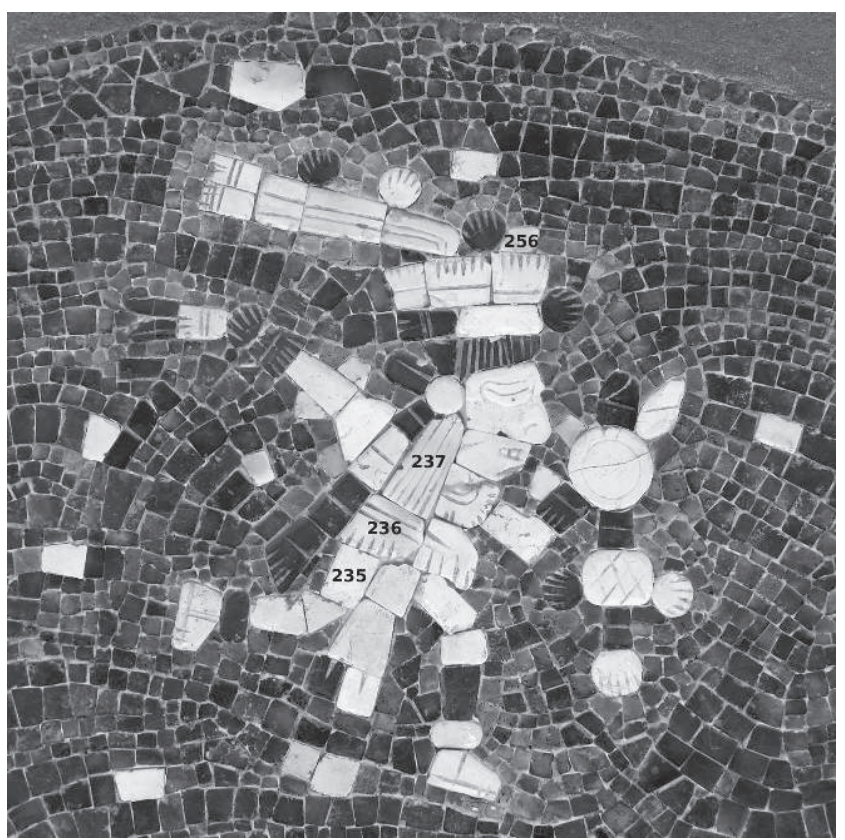

Figure 7 : Détail de l'offrande 99 avec, en clair, les tesselles analysées par XRF (53 mesures).

Figure 7: Detail of the offering 99. In light gray, the tesserae analyzed by XRF (53 measures).

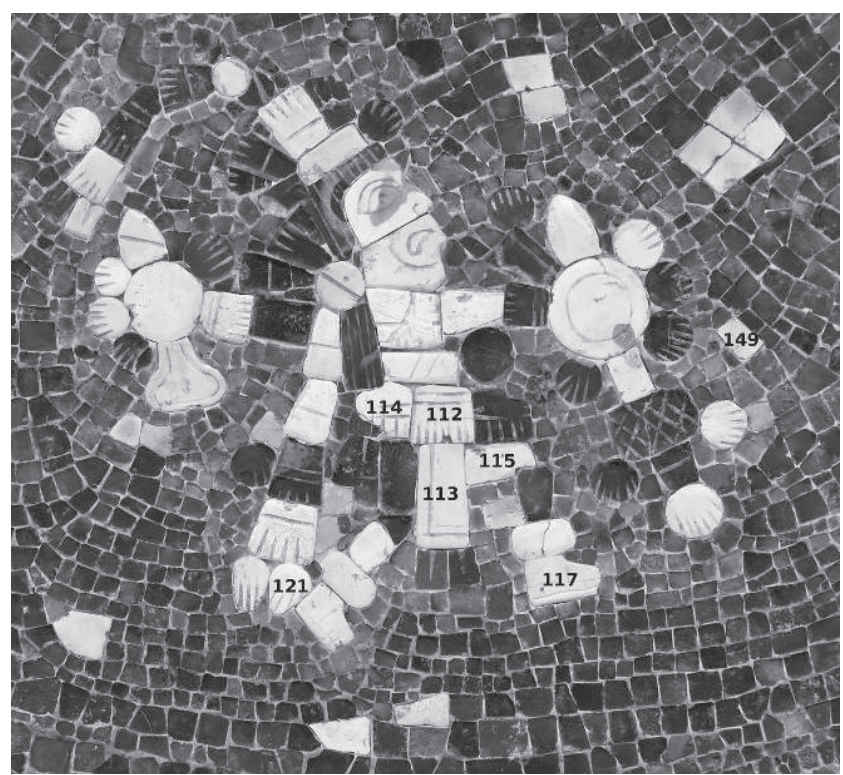

Figure 6 : Détail de l'offrande 99 avec, en clair, les tesselles analysées par XRF (55 mesures).

Figure 6: Detail of the offering 99. In light gray, the tesserae analyzed by XRF (55 measurements).

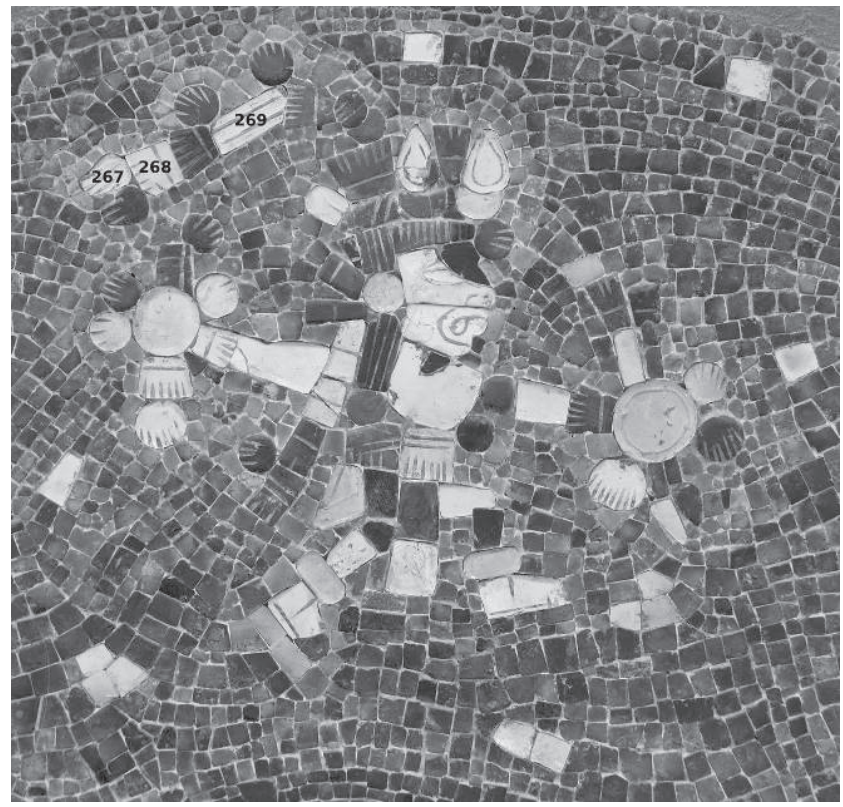

Figure 8 : Détail de l'offrande 99 avec, en clair, les tesselles analysées par XRF (50 mesures).

Figure 8: Detail of the offering 99. In light gray, the tesserae analyzed by XRF (50 measures). 


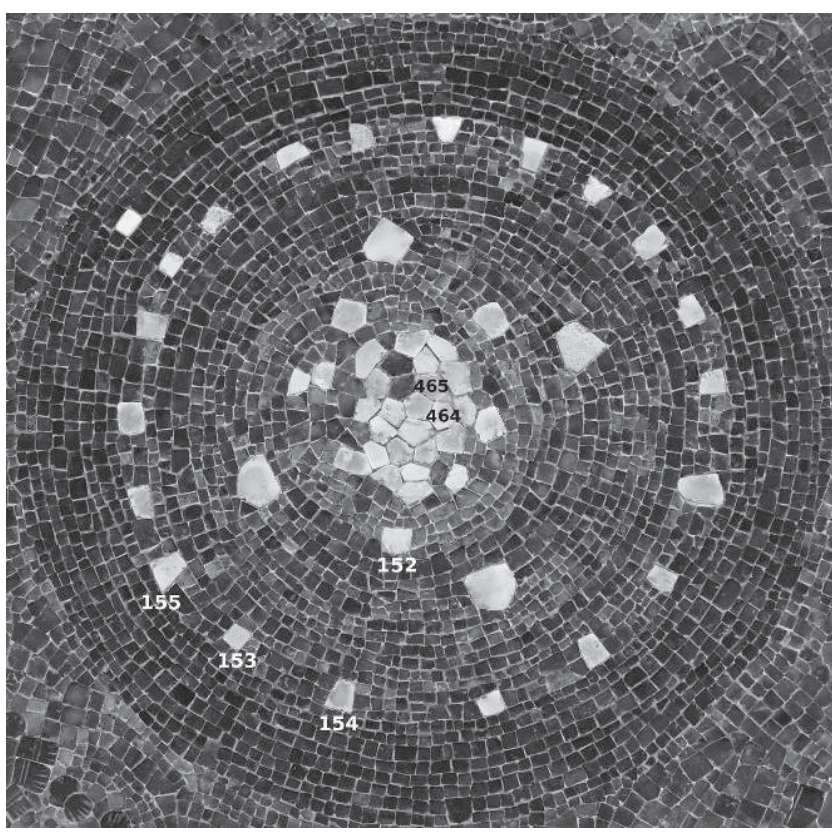

Figure 9 : Détail de l'offrande 99 avec, en clair, les tesselles analysées par XRF (49 mesures).

Figure 9: Detail of the offering 99. In light gray, the tesserae analyzed by XRF (49 measures).

En amont de cette étude, une série de photographies sous lumière ultraviolette (UV) permet de mettre en évidence les pierres présentant une fluorescence anormale vis-à-vis de l'ensemble de l'artefact. Quand elle ne révèle pas la présence d'une espèce minérale différente, cette fluorescence est liée généralement à la présence de matrice. Le temps d'analyse dont nous disposons au Musée du Templo Mayor est restreint. Dans le cas d'objets présentant un nombre très élevé de tesselles (comme l'offrande 99), nous analysons en priorité les plus grandes (entre 3 et $5 \mathrm{~mm}$ de côté). Dans un second temps, nous nous intéressons aux plus petites pierres présentant une fluorescence sous UV ou une couleur différente sous lumière naturelle. Ainsi, si des minéraux autres que des "turquoises minéralogiques " sont présents, ils devraient être surreprésentés dans l'échantillonnage. Le choix a été effectué de manière à obtenir une représentation la plus proche possible de l'objet en termes de tonalités de couleur, de tailles des tesselles, de localisations dans les personnages et dans le fond. La limite d'analyse réside essentiellement dans la taille du faisceau de rayons X $(1,5 \mathrm{~mm}$ de diamètre). Ainsi, nous prenons soin d'analyser les tesselles suffisamment grandes pour ne pas enregistrer la réponse spectrale de plusieurs tesselles simultanément. Les tesselles analysées par XRF se répartissent sur toute la surface du disque incluant des tesselles composant les sept personnages, ainsi que des tesselles composant le disque central et la bande extérieure du disque. Dans la bande intermédiaire plus claire, seulement une tesselle fût analysée (figure 9).

Les mesures s'effectuent toutes à la même distance de l'objet, grâce à deux lasers de position, de manière à obtenir des données uniformes et, ainsi, permettre leur comparaison. Les spectres XRF s'enregistrent avec le logiciel ADMCA (ADMCA Analog and Digital Acquisition, version 2.0.0.0, copyright (C) 1998-2010, Amptek Inc.). Ils sont ensuite dépouillés avec le logiciel WinQXAS (WinQXAS Quantitative X-ray Analysis System for MS Windows operating system, version 1.40, copyright (C) 2002 International Atomic Energy Agency) en utilisant une ligne de bruit de fond générée par un filtre digital de type "smooth filter » calculée avec vingt itérations. On conserve le même "ROI " (zone du spectre à partir duquel on modélise la ligne de bruit de fond) pour tous les spectres afin d'uniformiser les résultats. Bien que le logiciel calcule l'aire de tous les pics caractéristiques présents dans le spectre $(K \alpha, K \beta, L \alpha, L \beta$ et $L \gamma)$, seules les aires des pics $\mathrm{K} \alpha$ et $\mathrm{L} \alpha$ sont présentées dans les résultats. Les données sont normalisées et accompagnées de leurs erreurs statistiques (fournies par le logiciel WinQXAS).

\section{RÉsultats ET DisCUSSION}

À partir de la base de données minérale, nous avons défini le spectre XRF caractéristique des " turquoises minéralogiques » comme un spectre présentant les éléments $\mathrm{P}$ et $\mathrm{Cu}$ ainsi que les " éléments secondaires " caractéristiques du "Groupe Turquoise » : Fe et Zn. Idéalement, il ne doit pas apparaître de silicium.

Bien que, a priori, la distinction entre les " turquoises minéralogiques » et la chrysocolle soit possible à partir des spectres XRF de référence (figure 10), une limitation a été observée pour ces deux minéraux, au niveau de la distinction entre les turquoises et les chrysocolles archéologiques. Le phosphore et le silicium sont les deux éléments clés qui permettent de différencier les deux espèces minérales (tableau 3). Cependant, leurs quantités se trouvent parfois sous le seuil de détection de la XRF. D'autre part, le silicium peut provenir d'une contamination extérieure (argile du lieu d'enfouissement de l'artefact, par exemple). Ainsi, deux cas particuliers apparaissent dans les spectres XRF des tesselles archéologiques; on peut observer des spectres sans $\mathrm{P}$, ni $\mathrm{Si}$ et des spectres avec du Si et du P.

Dans un premier temps, nous avons décidé de définir comme "turquoises minéralogiques " uniquement les spectres présentant du $\mathrm{P}$ (tableau 5) et nous estimons que nous ne pouvons pas déterminer l'espèce minérale des tesselles dont les spectres XRF ne présentent ni silicium, ni 


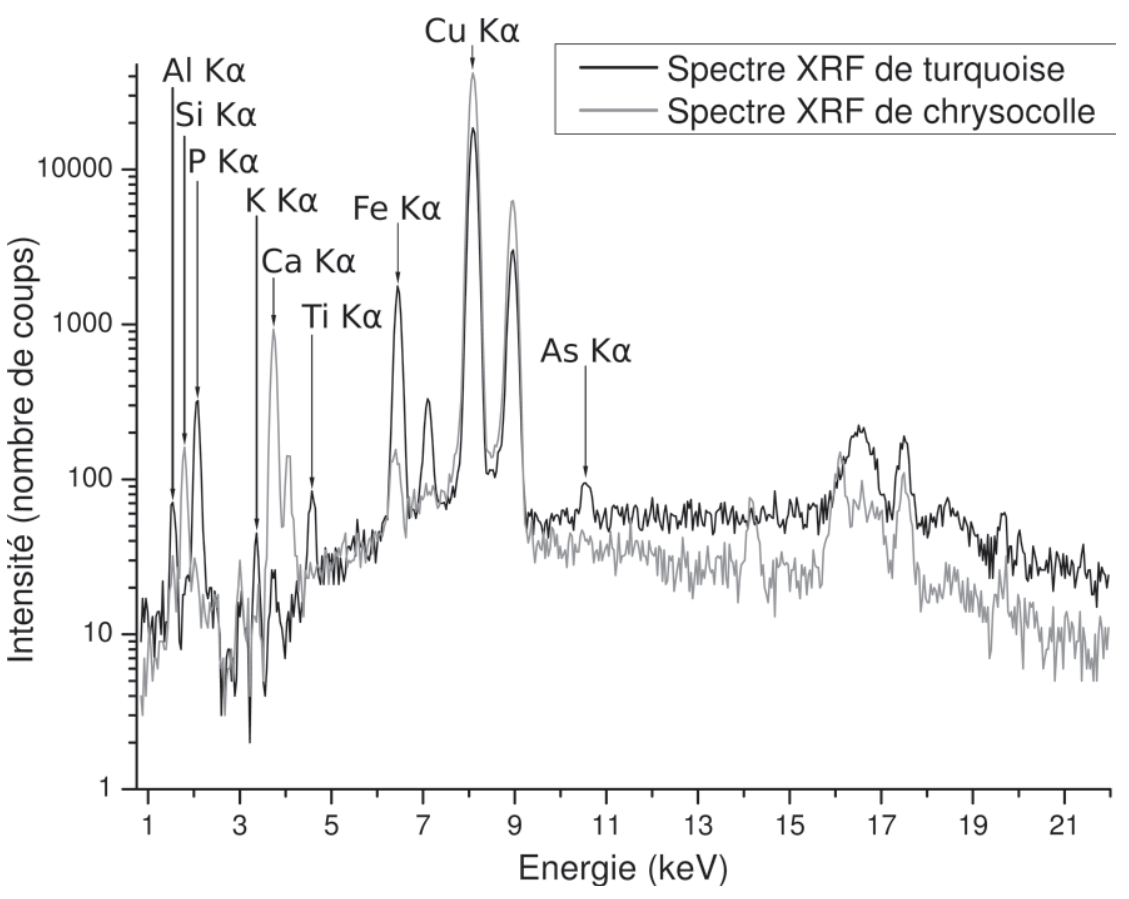

Figure 10 : Différences entre le spectre XRF d'une turquoise et celui d'une chrysocolle.

Figure 10: Differences between the XRF spectrum of a turquoise and the one of a chrysocolla.

\begin{tabular}{|l|c|c|c|c|c|c|c|c|c|c|}
\hline $\mathrm{No}$ & $\mathrm{Si}-\mathrm{K} \alpha$ & $\mathrm{P}-\mathrm{K} \alpha$ & $\mathrm{K}-\mathrm{K} \alpha$ & $\mathrm{Ca}-\mathrm{K} \alpha$ & $\mathrm{Mn}-\mathrm{K} \alpha$ & $\mathrm{Fe}-\mathrm{K} \alpha$ & $\mathrm{Cu}-\mathrm{K} \alpha$ & $\mathrm{Zn}-\mathrm{K} \alpha$ & $\mathrm{As}-\mathrm{K} \alpha$ & $\mathrm{Sr}-\mathrm{K} \alpha$ \\
\hline 112 & - & $0,63 \pm 0,06$ & - & $0,41 \pm 0,05$ & - & $18,5 \pm 0,2$ & $69,3 \pm 0,3$ & $11 \pm 0,2$ & $0,11 \pm 0,07$ & - \\
\hline 113 & - & $0,57 \pm 0,06$ & - & $0,51 \pm 0,05$ & - & $28 \pm 0,2$ & $59,5 \pm 0,3$ & $9,6 \pm 0,1$ & $1,53 \pm 0,08$ & $0,29 \pm 0,07$ \\
\hline 117 & - & $0,49 \pm 0,05$ & - & $0,39 \pm 0,05$ & - & $14,3 \pm 0,1$ & $74,4 \pm 0,3$ & $9,4 \pm 0,1$ & $0,71 \pm 0,08$ & $0,31 \pm 0,08$ \\
\hline 153 & - & $0,41 \pm 0,06$ & - & $0,44 \pm 0,05$ & - & $42,9 \pm 0,2$ & $51,9 \pm 0,3$ & $3 \pm 0,1$ & $1,15 \pm 0,07$ & $0,27 \pm 0,07$ \\
\hline 154 & - & $0,71 \pm 0,07$ & - & $1,67 \pm 0,08$ & $0,27 \pm 0,05$ & $1,18 \pm 0,08$ & $93,7 \pm 0,4$ & $1,7 \pm 0,1$ & $0,41 \pm 0,08$ & $0,35 \pm 0,09$ \\
\hline 235 & - & $0,74 \pm 0,09$ & - & $1,2 \pm 0,1$ & $0,92 \pm 0,09$ & $5 \pm 0,2$ & $78,8 \pm 0,5$ & $11,5 \pm 0,2$ & $1,7 \pm 0,2$ & - \\
\hline 237 & - & $0,53 \pm 0,08$ & - & $1,54 \pm 0,09$ & $0,3 \pm 0,05$ & $9,6 \pm 0,2$ & $83,7 \pm 0,4$ & $3,9 \pm 0,1$ & $0,3 \pm 0,1$ & - \\
\hline 267 & - & $0,44 \pm 0,07$ & - & $0,85 \pm 0,07$ & $0,38 \pm 0,05$ & $15,5 \pm 0,2$ & $76,5 \pm 0,4$ & $4,4 \pm 0,1$ & $1,59 \pm 0,09$ & $0,32 \pm 0,09$ \\
\hline 268 & - & $1,1 \pm 0,1$ & - & $1,7 \pm 0,1$ & $3,6 \pm 0,1$ & $9,1 \pm 0,2$ & $73,4 \pm 0,5$ & $9,4 \pm 0,2$ & $0,5 \pm 0,1$ & $0,7 \pm 0,2$ \\
\hline 348 & - & $0,52 \pm 0,05$ & - & $0,34 \pm 0,04$ & $0,15 \pm 0,04$ & $4,7 \pm 0,1$ & $57,8 \pm 0,3$ & $35,7 \pm 0,2$ & $0,86 \pm 0,08$ & - \\
\hline 349 & - & $0,69 \pm 0,06$ & - & $0,5 \pm 0,06$ & $0,15 \pm 0,04$ & $9,8 \pm 0,2$ & $75,4 \pm 0,4$ & $13,2 \pm 0,2$ & - & - \\
\hline 353 & - & $0,46 \pm 0,06$ & $0,34 \pm 0,06$ & $0,53 \pm 0,07$ & $0,44 \pm 0,05$ & $16,1 \pm 0,3$ & $65,7 \pm 0,3$ & $15 \pm 0,2$ & $1,37 \pm 0,09$ & - \\
\hline 372 & - & $0,54 \pm 0,07$ & $0,3 \pm 0,06$ & $0,84 \pm 0,08$ & $1,02 \pm 0,06$ & $16 \pm 0,3$ & $69,9 \pm 0,4$ & $10,8 \pm 0,2$ & $0,3 \pm 0,1$ & $0,4 \pm 0,1$ \\
\hline 373 & - & $0,64 \pm 0,06$ & - & $0,6 \pm 0,05$ & - & $42,3 \pm 0,2$ & $43,9 \pm 0,2$ & $12,2 \pm 0,1$ & - & $0,33 \pm 0,07$ \\
\hline
\end{tabular}

Tableau 5 : Aires des pics caractéristiques normalisées (avec leurs erreurs statistiques) de tesselles représentatives identifiées comme «turquoise minéralogique ".

Table 5: Normalized areas of the characteristic peaks (with their statistical errors) of tesserae identified as "chemical turquoise".

phosphore. Ce cas de figure concerne dix tesselles sur les 442 analysées pour l'offrande 99 (tableau 6). Dans ces cas précis, l'idéal serait de déterminer clairement l'espèce minérale en utilisant une autre technique d'analyse tel que la DRX portable en mode non destructif ou la spectroscopie Raman ou Infrarouge (FTIR) portable par exemple. Toutefois, à défaut de disposer d'un tel équipement, la XRF nous donne une première approximation rapide et efficace, pour un coût minime. Le second cas de figure (présence de Si et de P) concerne sept tesselles. La présence de Si peut provenir de la matrice d'argile humide remplissant l'offrande. Pour autant, la présence de $\mathrm{P}$ et la cohérence des "éléments secondaires " nous permettent de considérer, en seconde approximation, ces tesselles comme étant des " turquoises minéralogiques " 


\begin{tabular}{|l|c|c|c|c|c|c|c|c|c|c|}
\hline No & $\mathrm{Si}-\mathrm{K} \alpha$ & $\mathrm{P}-\mathrm{K} \alpha$ & $\mathrm{K}-\mathrm{K} \alpha$ & $\mathrm{Ca}-\mathrm{K} \alpha$ & $\mathrm{Mn}-\mathrm{K} \alpha$ & $\mathrm{Fe}-\mathrm{K} \alpha$ & $\mathrm{Cu}-\mathrm{K} \alpha$ & $\mathrm{Zn}-\mathrm{K} \alpha$ & $\mathrm{As}-\mathrm{K} \alpha$ & $\mathrm{Sr}-\mathrm{K} \alpha$ \\
\hline 121 & - & - & - & $0,7 \pm 0,06$ & - & $38,1 \pm 0,2$ & $50,7 \pm 0,3$ & $9,9 \pm 0,1$ & $0,22 \pm 0,07$ & $0,37 \pm 0,07$ \\
\hline 464 & - & - & $0,46 \pm 0,09$ & $2,9 \pm 0,2$ & $0,21 \pm 0,07$ & $10,9 \pm 0,3$ & $66,5 \pm 0,4$ & $17,3 \pm 0,3$ & $1,2 \pm 0,2$ & $0,5 \pm 0,2$ \\
\hline 465 & - & - & - & $0,85 \pm 0,07$ & $0,19 \pm 0,05$ & $10,9 \pm 0,2$ & $77,8 \pm 0,4$ & $10 \pm 0,2$ & $0,3 \pm 0,1$ & - \\
\hline 236 & - & - & $0,26 \pm 0,06$ & $1,28 \pm 0,09$ & $0,58 \pm 0,06$ & $9,4 \pm 0,1$ & $76,1 \pm 0,4$ & $12,1 \pm 0,2$ & $0,33 \pm 0,09$ & - \\
\hline 256 & - & - & - & $0,55 \pm 0,08$ & - & $7,5 \pm 0,1$ & $84,4 \pm 0,4$ & $5,3 \pm 0,2$ & $1,9 \pm 0,1$ & $0,3 \pm 0,1$ \\
\hline 269 & - & - & $0,57 \pm 0,08$ & $1,2 \pm 0,1$ & $0,47 \pm 0,06$ & $14 \pm 0,3$ & $77,5 \pm 0,4$ & $5,1 \pm 0,1$ & $1,2 \pm 0,1$ & - \\
\hline 411 & - & - & - & $1,28 \pm 0,08$ & - & $7,4 \pm 0,1$ & $81,5 \pm 0,4$ & $9,3 \pm 0,2$ & $0,46 \pm 0,09$ & - \\
\hline 412 & - & - & - & $1 \pm 0,07$ & - & $28,1 \pm 0,2$ & $60,4 \pm 0,3$ & $8,6 \pm 0,2$ & $1,8 \pm 0,1$ & - \\
\hline 413 & - & - & - & $2 \pm 0,1$ & $6 \pm 0,2$ & $9,5 \pm 0,2$ & $58,9 \pm 0,4$ & $22,3 \pm 0,3$ & $0,5 \pm 0,2$ & $0,7 \pm 0,2$ \\
\hline 422 & - & - & $0,41 \pm 0,08$ & $1,3 \pm 0,1$ & $0,31 \pm 0,07$ & $12,3 \pm 0,3$ & $77 \pm 0,5$ & $6,2 \pm 0,2$ & $2,5 \pm 0,2$ & - \\
\hline
\end{tabular}

Tableau 6 : Aires des pics caractéristiques normalisées (avec leurs erreurs statistiques) des dix tesselles ne présentant ni silicium, ni phosphore.

Table 6: Normalized areas of the characteristic peaks (with their statistical errors) of the ten tesserae that do not contain neither silicon nor phosphorus.

(tableau 7). La position des mesures présentées dans les tableaux 5, 6 et 7 sont indiqués sur les figures 4 à 9. Nous n'avons identifié aucune malachite, azurite, amazonite, ni lapiz lazuli. En conclusion, sur un total de 433 tesselles analysées, 424 peuvent être identifiées comme étant des «turquoises minéralogiques ", soit $98 \%$ des tesselles analysées. La confection de ce disque a été faite presque exclusivement à partir de "turquoises minéralogiques ". Cette observation implique une sélection précise ainsi qu'une connaissance poussée de la matière première employée, qu'il s'agisse de lieux d'approvisionnement spécifiques ou d'une aptitude à distinguer la turquoise des autres minéraux bleu-vert. Elle confirme l'importance de cette offrande et du message qu'elle véhicule. Il ne fait aucun doute que les offrandes étaient conçues pour permettre aux religieux de transmettre des messages précis aux dieux (Lopez Lujan, 1993; Duverger, 2007). La cosmovision aztèque, maîtrisée par les détenteurs du pouvoir et les prêtres, confère le pouvoir sémantique aux objets mais aussi à la matière première elle-même ou à sa provenance. L'imbrication de ces différents paramètres définit le message exact destiné aux divinités.

Dans le cas de la mosaïque de l'offrande 99 et comme nous avons pu l'observer dans toutes les autres offrandes de pierre bleue du Templo Mayor analysées (tableau 1), les " turquoises minéralogiques » jouent vraisemblablement un rôle important dans le signifiant de l'objet. Il est intéressant de préciser que la turquoise joue son rôle de signifiant par sa nature minérale et/ou sa provenance plutôt que par sa couleur (très variable).

Si l'emploi d'espèces minérales correspondantes au groupe des " turquoises culturelles " ne fait aucun doute, il semblerait que les Aztèques du Templo Mayor utilisaient exclusivement des "turquoises minéralogiques " pour confectionner leurs offrandes religieuses. Il serait justifié de parler d'erreur plutôt que d'acte intentionnel étant donné le pourcentage élevé de tesselles analysées définies comme "turquoises minéralogiques ». Au contraire, l'acte intentionnel résiderait plutôt dans une tentative d'emploi

\begin{tabular}{|l|c|c|c|c|c|c|c|c|c|c|}
\hline No $^{\prime}$ & $\mathrm{Si}-\mathrm{K} \alpha$ & $\mathrm{P}-\mathrm{K} \alpha$ & $\mathrm{K}-\mathrm{K} \alpha$ & $\mathrm{Ca}-\mathrm{K} \alpha$ & $\mathrm{Mn}-\mathrm{K} \alpha$ & $\mathrm{Fe}-\mathrm{K} \alpha$ & $\mathrm{Cu}-\mathrm{K} \alpha$ & $\mathrm{Zn}-\mathrm{K} \alpha$ & $\mathrm{As}-\mathrm{K} \alpha$ & $\mathrm{Sr}-\mathrm{K} \alpha$ \\
\hline 114 & $0,23 \pm 0,06$ & $0,37 \pm 0,06$ & - & $1,9 \pm 0,07$ & - & $37,6 \pm 0,2$ & $51,6 \pm 0,3$ & $6,7 \pm 0,1$ & $1,37 \pm 0,08$ & $0,24 \pm 0,07$ \\
\hline 115 & $0,27 \pm 0,06$ & $0,66 \pm 0,06$ & - & $0,88 \pm 0,06$ & $0,37 \pm 0,05$ & $8,7 \pm 0,2$ & $83,2 \pm 0,4$ & $5 \pm 0,1$ & $0,6 \pm 0,1$ & $0,31 \pm 0,1$ \\
\hline 149 & $0,25 \pm 0,08$ & $0,98 \pm 0,09$ & - & $1,22 \pm 0,09$ & $4 \pm 0,1$ & $5,7 \pm 0,2$ & $67,8 \pm 0,4$ & $18,8 \pm 0,3$ & $1 \pm 0,1$ & $0,2 \pm 0,1$ \\
\hline 152 & $0,2 \pm 0,05$ & $0,54 \pm 0,06$ & - & $0,37 \pm 0,05$ & - & $8,2 \pm 0,1$ & $65,9 \pm 0,3$ & $23,8 \pm 0,2$ & $0,95 \pm 0,08$ & $0,15 \pm 0,08$ \\
\hline 155 & $0,25 \pm 0,05$ & $0,62 \pm 0,06$ & - & $0,52 \pm 0,05$ & - & $9,1 \pm 0,1$ & $65,8 \pm 0,3$ & $22,2 \pm 0,2$ & $1,58 \pm 0,09$ & - \\
\hline 352 & $0,37 \pm 0,07$ & $0,4 \pm 0,07$ & $0,29 \pm 0,06$ & $1,44 \pm 0,09$ & $0,34 \pm 0,05$ & $45,4 \pm 0,4$ & $43,4 \pm 0,3$ & $6,4 \pm 0,1$ & $1,54 \pm 0,09$ & $0,3 \pm 0,08$ \\
\hline 374 & $0,26 \pm 0,06$ & $0,51 \pm 0,06$ & $0,35 \pm 0,06$ & $0,48 \pm 0,07$ & - & $17 \pm 0,2$ & $73,7 \pm 0,3$ & $5,7 \pm 0,1$ & $1,96 \pm 0,09$ & - \\
\hline
\end{tabular}

Tableau 7 : Aires des pics caractéristiques normalisées (avec leurs erreurs statistiques) des sept tesselles présentant du silicium. Table 7: Normalized areas of the characteristic peaks (with their statistical errors) of the seven tesserae containing silicon. 
exclusif de " turquoises minéralogiques " pour confectionner les objets d'offrande.

Les résultats obtenus pourraient être la preuve d'un approvisionnement en matière première brute qui aurait facilité l'identification de la nature du minéral par le lapidaire ou d'une préférence spécifique et contrôlée du lieu d'approvisionnement en matière première. Ces hypothèses sont appuyées par les travaux de tracéologie menés sur les tesselles (Melgar, 2010) qui mettent en évidence l'application d'une technologie typiquement mexica dans leur confection, situant l'élaboration de cet artefact dans les environs du Bassin de Mexico, voire dans l'enceinte même du centre cérémonial de México-Tenochtitlán. Ces hypothèses de travail permettent aux anthropologues et archéologues de repenser la chaine opératoire ainsi que les conséquences sur l'organisation du travail et les relations entre les zones d'approvisionnement en turquoise et la capitale aztèque (Wilcox et al., 2009).

Concernant les 10 tesselles non caractérisées, nous avons tenté d'observer si la localisation de ces tesselles présenterait une caractéristique particulière dans le disque : forme, couleur, attribut, ou partie de personnage. Après analyse, rien ne semble indiquer que ce soit le cas. Cependant, cette observation est limitée par le petit nombre de tesselles en question.

\section{Conclusion}

La présente étude révèle que les Mexicas réservaient une importance toute particulière à l'emploi quasi exclusif des "turquoises minéralogiques " dans la confection du disque de mosaïque de l'offrande 99 du Templo Mayor. Cette tendance à l'usage de la "turquoise " pour les mosaïques du Templo Mayor est confirmée par des études précédentes menées sur sept autres artefacts issus des fouilles archéologiques (Laclavetine, 2008). La proportion de « turquoises minéralogiques » sur l'ensemble des huit artefacts étudiés, représentant un total de 693 tesselles analysées, est de l'ordre de $95 \%$.

Ce travail permet de préciser l'usage des « turquoises culturelles » par le centre cérémoniel aztèque. C’est la première fois que cette problématique anthropologique est traitée par le biais de l'archéométrie. Les " turquoises minéralogiques " semblent tenir un rôle important dans le signifiant des objets d'offrande du Templo Mayor. Le fait que le signifiant soit lié à la nature minérale ou à sa provenance plutôt qu'à la couleur de la pierre est une hypothèse très intéressante qui nous oblige à réfléchir sur les connaissances que les Mexicas avaient acquises sur les minéraux bleus ou sur l'importance qu'ils pouvaient conférer aux lieux de provenance.
Si l'idée de " turquoises culturelles » met en évidence une variation d'usage des minéraux en fonction des époques et des régions, il ne faut pas la confondre avec un mélange des minéraux, dans un même lieu, pour un même objet. Il semblerait, dans le cas présent, que le contrôle sur l'emploi des "turquoises minéralogiques " fût très important dans le centre cérémoniel du Templo Mayor durant toutes les étapes de construction de l'édifice, soit une période de 80 ans. Malgré les fluctuations économiques, politiques, diplomatiques et les guerres ayant pu affecter la société mexica, tout au long de l'histoire aztèque, les objets d'offrande ont toujours été conçues à partir de "turquoises". Les lieux de provenance, la quantité et même la qualité de la matière première ne semblent pas être influencés par ces facteurs importants.

La chrysocolle, une fois travaillée en tesselles, présente un aspect similaire à la turquoise. Sa dureté est inférieure à celle de la turquoise (tableau 3), ce qui facilite le travail lors de la production de tesselles. Les mines de chrysocolle les plus proches se situent à environ $200 \mathrm{~km}$ de MéxicoTenochtitlán, dans les actuels états de Guerrero et de Morelos. $\mathrm{Au}$ contraire, la turquoise est plus difficile à travailler et les mines de turquoise, les plus proches connues, se situent à plus de $1000 \mathrm{~km}$ de la capitale aztèque. Cette observation confirme l'importance des "turquoises minéralogiques" dans la sémantique religieuse aztèque. La fonction de ces offrandes est d'ordre religieux, rituel et répond à une symbolique et un langage religieux fort, contrôlé et documenté (Lopez Lujan, 1993; Duverger, 2007).

Cette étude, originale dans le cas de la Mésoamérique et des Aztèques, est une première approximation pour distinguer les matériaux présents dans les contextes archéologiques. Elle est la première de cette nature à s'intéresser à la distinction des espèces minérales là où les études précédentes sur les " pierres bleu-vert " s'intéressaient directement à des études de provenance. Notre idée est de fournir une première approche concrète aux archéologues pour leur permettre de faire des études archéométriques viables sur des objets d'une très grande valeur pour nos sociétés contemporaines comme pour les sociétés antiques qu'ils étudient. Ce premier travail se limite à l'usage de la XRF, néanmoins, il est évident que notre méthodologie devra être compléter avec l'emploi d'autres techniques non destructives in situ comme la DRX portable, les spectroscopies IR (FTIR) ou Raman. D'autre part, il serait intéressant d'augmenter le corpus de tesselles analysées au Templo Mayor pour asseoir nos conclusions sur une base statistique encore plus forte ainsi que d'étudier d'autres sites mésoaméricains afin de déterminer l'expansion de cette pratique dans l'espace mais aussi dans le temps. 


\section{Remerciements}

Les auteurs remercient les techniciens de l'IFUNAM, pour leur précieuse collaboration lors des mesures par XRF : Karim Uriel López, Francisco Javier Jaimes, Juan Carlos Pineda, Margarito Vázquez et Antonio Ramírez. Nous souhaitons exprimer nos plus vifs remerciements aux responsables et aux personnels du Musée du Templo Mayor pour nous avoir procuré un accès privilégié aux artefacts et avoir veillé au bon déroulement de notre étude. Nous remercions le Professeur Jean-Claude Boulliard de la Collection de Minéraux de l'Université Pierre et Marie Curie pour avoir fourni les minéraux de référence. Les auteurs saluent les membres du Centre de Spectroscopie infrarouge du Muséum National d'Histoire Naturelle et son directeur, le Professeur François Fröhlich. Un grand merci au chimiste Rufino Lozano Santa Cruz et au technicien Santiago Aviles Puntos de l'Institut de Géologie de la UNAM pour leur collaboration. Un remerciement pour leurs corrections et suggestions à Anabelle Kriznar, Ziad Abou-Haidar et Chloé Laclavetine. Cette recherche a été réalisée avec le financement des projets du Mexique MOVIL I et II (CONACyT U49839-R et 131944) et du projet PAPIIT UNAM IN403210.

\section{Bibliographie}

Demortier G., 2005. Ion Beam Techniques for the Nondestructive Analysis of Archaeological Materials. In X-Rays for Archaeology. Springer-Verlag, Berlin, 67-100.

Duverger C., 2007. El primer mestizaje, La clave para entender el pasado mesoamericano. Taurus, Mexico.

Garrison E. G., 2003. Technique in Archaeological Geology. Springer-Verlag, Berlin, 215-230.

Harbottle G., Weigand P. C., 1992. Turquoise in PreColumbian America. Scientific American, 266(2) : 56-62.

Izeki M., 2008. Conceptualization of 'Xihuitl': History, Environment and Cultural Dynamics in Postclassic Mexica Cognition. British Archaeological Reports S1863.

Kim J., Simon A. W., Ripoche V., Mayer J. W., Wilkens B., 2003. Proton-induced $\mathrm{x}$-ray emission analysis of turquoise artefacts from Salado Platform Mound sites in the Tonto Basin of central Arizona. Measurement Science and Technology, 14 : 1579-1589.

Laclavetine K., Ruvalcaba-Sil J. L., Melgar E. Caracterización no destructiva in situ de turquesas del Templo Mayor de México-Tenochtitlan por XRF. In R. Esparza López, P. Weigand, M. Delgado (ed.). Nuevos aportes de las técnicas de arqueometría en el estudio y caracterización del Patrimonio Cultural. El Colegio de Michoacán, A.C., sous presse.
Laclavetine K., 2008. Caractérisation non-destructive in situ de turquoise d'offrandes du Templo Mayor de México-Tenochtitlán. Mémoire de Master 2, université Michel-de-Montaigne Bordeaux 3, France.

Lopez Lujan L., 1993. Las ofrendas del Templo Mayor de Tenochtitlan. INAH, Mexico.

Mathien F. J., Olinger B., 1992. Experiment with X-ray Fluorescence to determine trace element variability in turquoise composition. Archaeology, Art, and Anthropology : Papers in Honor of J.J. Brody: 123-134.

Mathien F. J., 2001. The organization of turquoise production and consumption by the prehistoric Chacoans. American Antiquity, 66 : 103-18.

Matos Moctezuma, E., 1994. The Great Temple of the Aztecs. Thames and Hudson, Londres.

Melgar Tisoc E. R., Solis Ciriaco R. B., 2010. Manufacturing Techniques of the Turquoise Mosaics from the Great Temple of Tenochtitlan, Mexico. In J.L. Ruvalcaba Sil, J. Reyes, J.A. Arenas, A. Velázquez (eds.). 2nd Latin-American Symposium on Physical and Chemical Methods in Archaeology, Art and Cultural Heritage Conservation (LASMAC 2009). Universidad Nacional Autónoma de México, Universidad Autónoma de Campeche, Instituto Nacional de Antropología e Historia, Mexico, 119-124.

Moens L., Von Bohlen A., Vandenabeele P., 2000. X-Ray Fluorescence. In Modern Analytical Methods in Art and Archaeology. Chemical Analysis Series, Volume 155, WileyInterscience, New York, 55-79.

Ronzio A. R., SAlmon M. L., 1967. Relation between sources and composition of turquoise. Journal of the Colorado-Wyoming, volume 5 .

Ruvalcaba Sil J. L., Bucio L., Marín M. E., Velázquez A., 2005. Estudio por XRD y haces de iones de teselas de un disco de turquesas del Templo Mayor de Tenochtitlán. In La Ciencia de Materiales y su Impacto en la Arqueología. Volumen 2. Academia Mexicana de Ciencia de Materiales. Lagares, Mexico, 95-111.

Ruvalcaba Sil J. L., Filloy L., Vaggi M., Tapia Gálvez L. H., SÁnchez Becerra R., 2010. Estudio no destructivo in situ de la Máscara de Malinaltepec. In La Máscara de Malinaltepec. CONACULTA-INAH, Mexico, 153-168.

Ruvalcaba J. L., Rámirez D., Aguilar V., Picazo F., 2010. SANDRA : A Portable XRF System for the Study of Mexican Cultural Heritage. X-ray Spectrometry, 39 : 338-345.

Ruvalcaba J. L., Melgar E., Calligaro T., 2011. Manufacturing Analysis and Non Destructive Characterisation of Green Stone Objects from the Tenochtitlan Templo Mayor Museum, Mexico. In I. Turbanti-Memmi (ed.). Proceedings of the 37th International Symposium on Archaeometry. Volume XLV. Springer-Verlag, Berlin, 299-304. 
SAlmon M. L., Ronzio A. R., 1962. An X-ray fluorescence analysis of turquoise. Journal of the Colorado-Wyoming, volume 4.

Saville M. H., 1922. Turquois Mosaic Art In Ancient Mexico. Museum of the American Indian Heye Foundation, New York.

Weigand P. C., Harbottle G., 1993. The role of turquoises in the ancient Mesoamerican trade structure. In The American Southwest and Mesoamerica: Prehistoric Exchange Systems in North America. Plenum Press, New York and London, 159-177.

Weigand P. C., Harbottle G., Sayre E. V., 1977. Turquoise sources and source areas in Mesoamerica and the southwestern USA. In Exchange Systems in Prehistory. Academic Press, New York, 15-34.
Wilcox D. R., Weigand P. C., Wood J. S., Howard J. B., 2009, Interacción cultural antigua del suroeste americano en el noroeste mexicano. El Colegio de Michoacan, Colección Ensayos, Zamora.

Zetina S., Ruvalcaba J. L., Falcón T., Hernández E., González C., Arroyo E., 2009. Painting Syncretism : a Non Destructive Analysis of the Badiano Codex. From [http:// www.ndt.net/search/docs.php3?MainSource=65], consulté en 07/2011. 